\title{
Evaluación de la salud mental de personas mayores, víctimas del conflicto armado, atendidas en el Centro de Orientación Sociojurídica de Sincelejo en los años 2012-2013.
}

\section{Mental health evaluation of older people, victims of the armed conflict, attended at the Counseling Center Sociojurídica of Sincelejo in the years 2012-2013}

\author{
Sandra Castillo-Santis ${ }^{1}$ Psicól, Kelly Romero-Acosta ${ }^{12}$ Ph.D, Marta Sahagún-Navarro ${ }^{13}$ M.Sc \\ Andrea del Carmen Sánchez-Vergara ${ }^{1}$ Psicól.
}

${ }^{1}$ Corporación Universitaria del Caribe. Sincelejo - Colombia. ${ }^{2}$ Dra. en Psicopatología de niños, adolescentes y personas. Docente-investigadora. ${ }^{3} \mathrm{Mg}$. en Atención sociosanitaria de personas mayores y personas con discapacidad. Docente-investigadora.

Recibido: 16-01-2017; Aceptado: 27-03-2017

\section{RESUMEN}

El objetivo de la presente investigación es describir el estado de salud mental de las personas mayores, víctimas del conflicto armado, que visitaron el Centro de Orientación Sociojurídica (COS) de Sincelejo en los años 2012-2013. En total, participaron 14 víctimas cuyas edades oscilan entre los 60 y 82 años. De estas, dos eran hombres $(14,28 \%)$ y el resto, mujeres. Se les realizó la historia clínica psicológica, la cual detalla datos generales, motivo de consulta, examen mental, historia personal y familiar, observaciones, diagnóstico, plan de tratamiento y recomendaciones. Para esta investigación, solo se tuvo en cuenta el examen mental, en el cual la mayoría mostró actitud y apariencia de tristeza y preocupación, con buena orientación en tiempo, lugar y espacio, sin alteraciones en el lenguaje y percepción. Solo 2 personas presentaron pérdida de la memoria, y 4 se notaron con atención dispersa; 2 presentaron dificultades en la movilidad; el lenguaje fue fluido y coherente, con pensamiento lógico. Y en cuanto a la efectividad, 8 de ellas se notaron tristes $y / 0$ angustiadas. Se concluye entonces que el haber sido víctima del conflicto armado, ya sea por causa del desplazamiento forzado cuya experiencia implica la pérdida parcial o total de bienes materiales o la pérdida de familiares, es un desencadenante a la hora de padecer trastorno depresivo y/o ansioso en las personas mayores.

Palabras clave: Personas mayores, examen mental, conflicto armado, víctimas, salud mental. 
Castillo-Santis et al - Evaluación de la salud mental de personas víctimas del conflicto armado

\section{ABSTRACT}

The aim of this research is to describe the mental examination of elderly victims of armed conflict who visited the Socio-Legal Counseling Center (COS) of Sincelejo in the years 2012-2013. In all, 14 victims whose ages of 60 and 82 years. Of these, two were men (14.28\%) and the rest women. They underwent psychological history, which is comprised of general data, reason for consultation, mental examination, personal and family history, observations, diagnosis, treatment plan and recommendations; after made, we continued with the analysis; it should be noted that for this investigation only the mental examination, which it was noted that most showed attitude and appearance of sadness and worry, with good orientation in time, place and space, without alterations in language and perception are taken into account. Only 2 people had memory loss and 4 lawere noted with scattered attention, 2 presented problems for the movement itself, the language was fluid and consistent with logical thinking. As for effectiveness, 8 of them were noted sad and / or distressed. Indicating that they had been victims of the armed conflict either because of forced displacement whose experience involves the partial or total loss of property or the loss of family is a trigger when suffering from depressive disorder and / or anxious in older adults.

Keywords: Elderly, mental examination, armed conflict, victims, mental health.

\section{INTRODUCCIÓN}

La Ley 1448 de 2011 o la Ley de Víctimas y Restitución de Tierras, en su artículo $3^{\circ}$, considera víctima a:

Toda aquella persona que ha sufrido pérdida de sus derechos a partir de 1985, como consecuencia de infracciones al Derecho Internacional Humanitario o de violaciones graves a las Normas Internacionales de Derechos Humanos. También, se considera víctimas a los familiares en primer grado de consanguinidad y a los cónyuges de los afectados. De la misma forma, se consideran víctimas a las personas que han sufrido un daño al intervenir para asistir a la víctima en peligro o para prevenir la victimización.

La guerra constituye un acontecimiento de extraordinaria intensidad, repentino, dramático, imprevisible y fuera del control personal, ya que puede llevar al desarraigo personal, la pérdida, la separación, la desintegración familiar, el sufrimiento mental y físico y el cambio social sustancial. Las personas que han vivido sometidas a los rigores de la guerra sufren trastornos emocionales que reflejan la dificultad del ser humano por adaptarse a la violencia. Las víctimas de la violencia tienen un mayor riesgo de desarrollar graves problemas de salud mental, en especial las personas mayores y de la tercera edad (Saunder et al, 2009; Kilpatrick y Resnick, 2007). Se debe tener en cuenta que el envejecimiento, o etapa de senectud, es la última etapa del ciclo de vida, en el cual el cuerpo manifiesta una "disminución cognitiva y fisiológica, causando aislamiento y la pérdida de autonomía e independencia, porque el rendimiento físico y social de las personas mayores depende de la integridad de todas sus funciones" (Marinês, Castioni, Kirchner y Hildebrandt, 2015, p. 3).

Este periodo del ciclo de vida es inevitable y cada vez más, esta población es mayor en muchos países latinoamericanos, tal como lo indica Dueñas (2011): "la Organización Iberoamericana de Salud Mental (OISS) calcula que en 2050, en América Latina habrá 136 millones de personas mayores; hoy son aproximadamente 100 millones, y hay tantos mayores de 60 años, como niños menores de cinco años" (p. 70). 
Las cifras anteriores son realmente alarmantes, pues las personas mayores pierden la capacidad funcional de realizar las actividades cotidianas, entendiéndose como funcionalidad "la capacidad que tiene un individuo de realizar sus actividades básicas cotidianas de forma independiente" (OMS, 2009, citado en Cardona, Estrada, Segura, Chavarriaga, Ordóñez y Osorio, 2008, p. 4).

Asimismo, "las personas mayores no se encuentran muchas veces con los recursos personales idóneos para adaptarse a situaciones y nuevas maneras de ver la realidad y de actuar en ella" (Bermejo, 2010, citado en Fernández et al, 2014, p. 522), lo que conduce a determinar que la población mayor atraviesa una serie de cambios psicológicos, sociales y fisiológicos que requieren una atención específica.

Los cambios más notorios que surgen en la etapa de la vejez son los fisiológicos, ya que es durante ella cuando se manifiestan todas las transformaciones que sufre el cuerpo, tal como el declive en los sentidos (gusto, oído, tacto, vista y olfato), la alteración del aspecto corporal (piel, masa muscular, peso...), así como los cambios en los órganos, sistemas y aparatos biológicos.

De igual forma, los cambios psicológicos son evidentes en cuanto a la eficacia de la realización de actividades de la vida diaria (AVD), así como en las actividades instrumentales de la vida diaria (AIVD), ya que es en este aspecto en el que surgen cambios cognitivos, y entre los más importantes, se encuentran las transformaciones en la memoria, el procesamiento de la información y la percepción que tengan las personas mayores de sí mismas, lo que influirá en su motivación y en sus relaciones interpersonales, tal como es descrito por Dorantes, Ávila, Mejía y Gutiérrez (2001, citado en Melguizo, Acosta y Castellano, 2012) quienes informaron que:
La mayor edad, padecer de enfermedad cerebrovascular, un mayor número de enfermedades crónicas, síntomas depresivos, deficiencia visual, dolores que limitan sus actividades diarias y tener algún miembro amputado resultaron ser factores asociados con la dependencia para realizar actividades instrumentales de la vida diaria ( $p$. 260).

En el caso de la memoria de trabajo García y colaboradores (2014, p. 337) hicieron referencia a que "las personas mayores muestran una mayor dificultad para realizar tareas que exigen mayor atención, flexibilidad mental, planificacióny procesos dereorganización del material" (Hernández-Ramos y Cansino, 2011; Pousada, de la Fuente, Gómez, y Armadans, 2004; Thornton y Raz, 2006; Verhaeghen y Basak, 2005).

Por otra parte, en el caso de la memoria episódica "el deterioro aparece fundamentalmente en: a) tareas que implican un esfuerzo de codificación y recuperación de la información importante" (Belleville et al, 2006; Clarys et al, 2007; Souchay, Isingrini, y Espagnet, 2000; citado en García et al, 2014, p. 338); "y b) el recuerdo de hechos recientes o prospectivos ligados a los acontecimientos de la vida diaria del sujeto: recordar nombres de personas, números de teléfono, dónde han dejado sus objetos, etc." (Montejo, y Montenegro, 2006; citado en García et al, 2014, p. 338).

Los más afectados por los mitos sobre la avanzada edad son los propios mayores, llegando en muchos casos a identificarse con esas creencias extendidas, y las consecuencias no son triviales. Igual que sucede en otras situaciones, aceptar ciertas ideas favorece que se conviertan en realidad (Gómez, Vázquez y Hervás, 2010; citado en Flecha, 2015, p. 322).

Cada vez es mayorel número de personas que, por diversos motivos, viven solas, sin nadie que pueda responder a sus 
preguntas, que tome en serio sus problemas; sin nadie a quien preguntar o a quien dirigirse como un tú personal, que escuche y comprenda, que acoja y estimule; sin nadie con quien reír o llorar $y$, en definitiva, sin nadie con quien vivir. Este es, quizás, el lado más oscuro, desesperanzador y negativo de la existencia humana (Tamayo, 2001; citado en Limón y Crespo, p. 115).

La función cognitiva, el estado físico y la estabilidad social son los aspectos más influyentes en la capacidad funcional de los ancianos; estos aspectos están directamente relacionados con la calidad de vida, y, en ocasiones, son predictores del riesgo de fragilidad o de necesidades no cubiertas en las personas de edad avanzada (Corrales, Palomo, Magariño, Alonso y Torrico, 2004; citado en Villareal y Month, 2012, p. 84).

Por tanto, la sociedad se ha visto más preocupada por la integridad de las personas mayores, tomando medidas que faciliten el acceso de esta población a la educación y programas de intervención acompañados de la creación de políticas públicas encargadas de velar por el cumplimiento de los derechos de las personas de la tercera edad.

En este sentido, existen programas de formación universitaria como medio de intervención eficaz que no solo trae consigo beneficios educativos para las personas mayores, sino también para las personas que interactúan con ellas, tal y como lo expresa el Libro Blanco sobre Envejecimiento Activo (IMSERSO, 2011, citado en Fernández et al, 2014):

Los Programas Universitarios para Mayores (PUM) (...) poseen una gran eficacia simbólica por la presencia de las personas mayores en la Universidad; permiten la convivencia y el encuentro intergeneracional; favorecen el desarrollo del asociacionismo y del intercambio entre alumnos de universidades de otras comunidades autónomas y otros países; permiten abrirse a oportunidades en experiencias de aprendizaje más complejas (como las TIC) y participar en investigaciones y en proyectos de carácter europeo. Además es muy importante valorar los esfuerzos de estos programas por mejorar sus procesos de enseñanza-aprendizaje ( $p$. 526).

Es significativo destacar que la Declaración Mundial sobre la Educación Superior apoya este tipo de medidas al establecer "como una acción prioritaria, que el acceso a los establecimientos de educación superior debe abrirse a los educandos adultos, creando oportunidades de aprendizaje de manera flexible, abierta y creativa" (UNESCO, 2010; citado en Fernández et al, 2014, p. 527).

Asimismo, en la I Asamblea Mundial del Envejecimiento, el Plan de Viena (1982) "reconoce, por primera vez, la dimensión educativa en la vejez y la importancia del desarrollo de la educación con el objetivo de obtener una mejor calidad de vida" (citado en Fernández et al, 2014, p. 524), de manera que en la II Asamblea Mundial del Envejecimiento, celebrada en Madrid (2002), se estableció como "propósito guiar la formulación de políticas hacia el objetivo de lograr durante toda la vida y en toda la sociedad el ajuste social, cultural y económico a un mundo que envejece" (citado en Fernández et al, 2014, p. 524).

Por otra parte, la Organización Iberoamericana de la Seguridad Social (OISS; citado en Dueñas, 2011) indica "tres bloques de derechos necesarios para la persona mayor: salud, pensiones y servicios sociales. Cuestión que es relevante porque plantea tres bloques preferentes para defender a la persona mayor; eso implica que hay un mayor escudo de protección" (p. 71).

Esto permite reconocer que sí hay políticas que velen por el bienestar 
integral de las personas mayores, ampliando el abanico de opciones que den acceso a elegir la mejor forma de promover, entre esta población, una calidad de vida adecuada durante su vejez.

La efectividad de las políticas públicas estará en la integralidad que se logre entre los sectores de protección, seguridad social, salud, trabajo, medio ambiente, alimentación y nutrición y en el seguimiento, evaluación del alcance e impactos de los planes, programas y proyectos (Bejarano, Ardila y Montaño, 2014, p. 73).

En primera instancia están "los programas que se fundamentan en los principios de neuroplasticidad cerebral, reserva cognitiva y en la capacidad de aprendizaje" (Calero y Navarro, 2006b; Fernández Ballesteros, 2009; citado en García et al, 2014, p. 338), y se centran en dos estrategias de entrenamiento: a) "entrenar tan solo alguno de los factores implicados en el buen funcionamiento de la memoria enfoque que recibe el nombre de entrenamiento unifactorial" (García, García y Rodríguez, 2011; citado en García et al, 2014, p. 338) y b) "entrenar varios de los factores que influyen en la memoria y/o diversas estrategias cognitivas que recibe el nombre de entrenamiento multifactorial (Stigsdotter y Bäckman, 1995; citado en García et al, 2014, p. 338).

Otra estrategia de intervención son los programas de entrenamiento mnemotécnico que, a su vez, son los más utilizados, es decir, "programas destinados a entrenar el aprendizaje de diversas estrategias y técnicas de memoria (visualización, asociación, categorización, método loci, método de la cadena, etc.) que después puedan aplicarse 0 transferirse a situaciones concretas de su vida cotidiana" (García et al, 2014, p. 338).

Un tipo de modelo utilizado con menor frecuencia, pero que posee una eficacia significativa, son los que "se llevan a cabo a través de un entrenamiento centrado en olvidos cotidianos" (Martin y Kaiser, 1998; citado en García et al, 2014, p. 338). En este caso, "los módulos de entrenamiento del programa se diseñan para entrenar los olvidos más frecuentes que suelen presentar las personas mayores con quejas subjetivas de memoria y no tanto en el entrenamiento de estrategias de memoria" (García et al, 2014, p. 338).

De esta manera, queda reflejado que "el envejecimiento activo constituye, en definitiva, un aspecto fundamental para combatir y demorar situaciones de dependencia tanto físicas, como psíquicas, sociales, educacionales y funcionales" (IMSERSO, 2011; citado en Fernández et al, 2014, p. 524) que se acompañan de diversas estrategias que le suministran a las personas mayores las herramientas necesarias para su mayor bienestar y/o calidad de vida. Tal y como afirma Tonon de Toscano (2010, citado en Flecha, 2015):

La calidad de vida implica la participación de los ciudadanos en la evaluación de lo que les afecta, e incluye el estudio del bienestar físico y psicológico de las personas, relacionando las necesidades materiales con las socio- afectivas e integrando mediciones psicológicas y sociales de percepción y evaluación de las propias experiencias de los sujetos (p. 323).

De lo que se deduce que todos los ciudadanos hacen parte del cambio social, y un aspecto importante de ello es lograr que las personas mayores sean autosuficientes, con la finalidad de proporcionarles mayor bienestar $y / o$ calidad de vida para cada una de sus necesidades, tanto materiales como fisiológicas, psicológicas y sociales.

Esta investigación parte de la necesidad de ahondar en estudios que relacionen las variables de salud mental en personas mayores, víctimas del conflicto armado, 
pues son muy pocos los estudios publicados que existen. Ello se evidencia en una búsqueda exhaustiva realizada en octubre de 2015 en diferentes bases de datos, utilizando como palabras clave: personas mayores y conflicto armado, en ellas el número de artículos hallados fue muy bajo (exactamente once).

Otros autores como Campo-Arias, Oviedo y Herazo (2014), tras una revisión de artículos publicados en los buscadores de Google Académico, Bireme y PubMed desde 1994 hasta 2013, utilizando como palabras clave conflicto armado, trastornos mentales, salud mental, prevalencia y Colombia; identificaron que efectivamente, son muy pocos los estudios que analizan estas variables. De hecho, no encontraron estudios epidemiológicos previos al año 2000. Sin embargo, los pocos estudios hallados arrojaron información sobre la alta frecuencia de ansiedad y depresión relacionada con factores estresantes como, por ejemplo, el estrés postraumático.

\section{METODOLOGÍA}

Con enfoque cuantitativo y alcance descriptivo, este es un estudio de base documental de carácter retrospectivo.

Población y muestra: Este estudio se llevó a cabo en Sincelejo, una ciudad colombiana que pertenece al departamento de Sucre y que tiene una población de 267.571 habitantes, según el último estudio estadístico del DANE (2012). De esta ciudad, se eligió de forma intencionada el Centro de Orientación Sociojurídica (COS) de Sincelejo, el cual se encarga de brindar la atención y el acompañamiento psicosocial y jurídico a las víctimas del conflicto armado de todos los géneros, clases sociales y edades. De igual forma, este implementa un modelo de atención sistémica e integral, mediante un análisis crítico, reflexivo e investigativo de sus necesidades y realidades, promueve alianzas con otros actores sociales de orden local, nacional e internacional.

En el transcurso de los años 2012 y 2013 se atendieron en el área de psicología a 14 personas mayores que habían sido víctimas del conflicto, once del año 2012 y tres, del 2013. En este período se realizaba el examen mental a los visitantes.

Cuando se habla de examen mental, se hace referencia a la técnica que se utiliza para tener un diagnóstico más preciso sobre los trastornos o síndromes presentados por las personas atendidas (López y Giuliani, 2012). El examen mental está conformado por: actitud, apariencia, orientación, memoria, atención, motricidad, afecto, lenguaje y pensamiento. Luego de realizar el examen mental, se hizo un posible diagnóstico, el cual fue tomado de la descripción de la problemática y el motivo de consulta dado por la persona.

Cabe destacar que a estas catorce personas se les diligenció la historia clínica psicológica, la cual está conformada por datos generales, motivo de consulta, examen mental, historia personal y familiar, observaciones, diagnóstico, plan de tratamiento $y$ recomendaciones.

Procedimiento: El análisis de los datos se realizó con la ayuda del Statistical Package for Social Science (SPSS, V 20). En este análisis se calcularon frecuencias y porcentajes.

\section{RESULTADOS}

Información sociodemográfica. Las edades de los participantes oscilan entre 60 y 82 años. De esta muestra, dos son hombres $(14,3 \%)$ y el resto, mujeres ( $85.3 \%)$. Todas las mujeres se dedican a las labores del hogar, y los hombres a la albañilería. En la tabla 1 se muestra de manera más detallada esta información. 
Tabla 1. Datos sociodemográficos.

\begin{tabular}{ccc}
\hline Sexo & N & $\%$ \\
\hline Mujeres & 12 & $85.7 \%$ \\
Hombres & 2 & $14.3 \%$ \\
Edad & & \\
60 & 2 & $14.3 \%$ \\
62 & 2 & $14.3 \%$ \\
64 & 1 & $7.1 \%$ \\
67 & 1 & $7.1 \%$ \\
68 & 1 & $7.1 \%$ \\
70 & 3 & $21.4 \%$ \\
76 & 2 & $14.3 \%$ \\
80 & 1 & $7.1 \%$ \\
82 & 1 & $7.1 \%$ \\
Ocupación & & \\
Ama de casa & 12 & $85.7 \%$ \\
Maestro de obra & 2 & $14.3 \%$ \\
\hline
\end{tabular}

Fuente: Elaboración propia

Evaluación del paciente. La mayoría de las víctimas mostraron una actitud desanimada, con rasgos de tristeza, melancolía, preocupación y ansiedad. Con respecto a la apariencia, 8 de las víctimas (57.14\%) mostraron una apariencia de tristeza, desconsuelo, angustia y melancolía. De hecho, en la valoración de la afectividad, once de las víctimas se mostraron tristes, angustiados y ansiosos. Cabe destacar que todos mostraron una orientación adecuada y fluidez y coherencia en el lenguaje. Toda esta información se puede ver de manera más detallada en la tabla 2.

\section{DISCUSIÓN}

"La edad suele tener una serie de efectos en el funcionamiento y en el rendimiento cognitivo, tales como un enlentecimiento en el procesamiento de la información" (Brigman y Cherry, 2002; Myerson, Hale, Swagstaff, Poon, y Smith, 1990; Salthouse, 1996, citado en García, Fernández, Fuentes, López y Moreno, 2014, p. 337), lo que va acompañado de un descenso en los diferentes tipos de memoria. En este sentido, se identifica que "el establecimiento de una pérdida de memoria asociada al
Tabla 2. Evaluación del estado mental

\begin{tabular}{|c|c|c|}
\hline Actitud & $\mathbf{n}$ & $\%$ \\
\hline Desánimo, tristeza, preocupación & 9 & $64.3 \%$ \\
\hline Disponible, alerta & 4 & $28.6 \%$ \\
\hline Disponible-triste & 1 & $7.1 \%$ \\
\hline \multicolumn{3}{|l|}{ Apariencia } \\
\hline Tristeza, desconsolación, angustia y melancolía & 8 & $57.1 \%$ \\
\hline No registran datos & 6 & $42.9 \%$ \\
\hline \multicolumn{3}{|l|}{ Orientación } \\
\hline Orientación adecuada & 14 & $100 \%$ \\
\hline \multicolumn{3}{|l|}{ Memoria } \\
\hline Eumnésico & 9 & $64.3 \%$ \\
\hline Pérdida de algún tipo de memoria a largo plazo. & 2 & $14.3 \%$ \\
\hline Pérdida de algún tipo de memoria mediano plazo & 3 & $21.4 \%$ \\
\hline \multicolumn{3}{|l|}{ Atención } \\
\hline Euproséxico & 8 & $57.1 \%$ \\
\hline Atención dispersa. & 4 & $28.6 \%$ \\
\hline Sin registro. & 2 & $14.3 \%$ \\
\hline \multicolumn{3}{|l|}{ Motricidad } \\
\hline Por sus propios medios & 9 & $64.3 \%$ \\
\hline Con alguna ayuda & 2 & $14.3 \%$ \\
\hline Sin registro & 3 & $21.4 \%$ \\
\hline \multicolumn{3}{|l|}{ Afectividad } \\
\hline Tristeza, angustia y ansiedad & 11 & $78.6 \%$ \\
\hline Tranquilo y/o estable & 3 & $21.4 \%$ \\
\hline \multicolumn{3}{|l|}{ Lenguaje } \\
\hline Fluido y coherente & 14 & $100 \%$ \\
\hline \multicolumn{3}{|l|}{ Pensamiento } \\
\hline Lógico, coherente y racional & 12 & 85.7 \\
\hline Pensamiento distorsionado Sin registro & 1 & $7.1 \%$ \\
\hline
\end{tabular}

Nota: Elaboración propia

envejecimiento, de carácter benigno, puede llegar a provocar en los individuos que la presentan, ciertas dificultades para afrontar algunas tareas de la vida diaria" (García et al, 2014, p. 337).

No obstante, la memoria sensorial, inmediata, procedimental y el rendimiento entareas de reconocimiento, suelen estar preservados en este tipo de problemáticas (Kester, Benjamín, Castel y Craik, 2002; Spencer y Raz, 1995; citado en García et al, 2014). 
"Por el contrario, la memoria operativa y la memoria episódica sí presentan claras alteraciones" (García et al, 2014, p. 337).

En este estudio, dos personas tuvieron pérdida de memoria a largo plazo, y tres a mediano plazo. La pérdida de memoria de estos adultos mayores pudo deberse a efectos de la edad y no a efectos de hechos traumáticos por la guerra.

Todas estas deficiencias cognitivas pueden llegar a provocar en las personas mayores sentimientos de inferioridad e inutilidad, por lo que no pierde relevancia mencionar que la percepción que tengan de sí mismas es un punto clave para obtener un envejecimiento activo en esta población, tal como es descrito por Pérez (2006, citado en Flecha, 2015) el cual expresa: "que la imagen negativa que las personas mayores tienen sobre sí mismas funciona como un freno para su envejecimiento activo y saludable" (p. 322).

La auto-percepción del adulto mayor en el marco del conflicto puede ser en alto grado negativa debido a las situaciones de pérdida y dolor experimentadas. Luego de analizar el examen mental realizado en el Centro de Orientación Sociojurídica de Sincelejo, se pudo concluir que la mayoría de las víctimas mostraron una actitud, apariencia y afectividad de tristeza, angustia y preocupación.

Ello permite deducir que el haber sido víctima de manera directa o indirecta del conflicto armado es un factor predominante a la hora de padecer de trastorno depresivo y/o ansioso en las personas mayores, ya sea mediante el desplazamiento forzado, que ocasiona la pérdida parcial o total de bienes materiales, de igual manera, el asesinato o desaparición de familiares, que afecta la actitud, apariencia y afectividad de las personas, entre otros hechos victimizantes.
En efecto, Tonon de Toscazo (2010) afirma que el bienestar psicológico de las personas depende en gran medida de la valoración que cada persona hace de su situación conforme a su experiencia. Esta percepción incluye medidas tanto positivas como negativas, y una visión global de la vida a la que el autor denomina satisfacción vital. Todo esto permite determinar que las personas mayores recrean su vejez, de acuerdo con las experiencias.

Asimismo, es necesario hacer énfasis en que cada vez más, muchas de estas personas mayores atraviesan situaciones precarias de alimentación, vivienda $y$, en algunas ocasiones, de afecto. Ahora bien, si los adultos mayores que visitaron el COS, no solo tuvieron experiencias traumáticas, sino también, vivían en situaciones precarias de alimentación y vivienda al momento de contestar las preguntas de la historia clínica, la percepción sobre su situación sería mucho más negativa.

Actualmente, muchas entidades están tomando las medidas preventivas necesarias para evitar consecuencias psicológicas, sociales y fisiológicas en la población mayor, a través de la promoción de acciones decisivas que provoquen la inserción social, el envejecimiento activo $y$, por supuesto, una calidad de vida óptima.

Estas acciones sobre adultos mayores víctimas del conflicto serían de gran utilidad porque mejoraría su calidad de vida teniendo en cuenta, tanto las consecuencias normales del proceso de envejecimiento del adulto mayor, como las relacionadas con las vivencias de situaciones traumáticas. Porque aquí no solo se trata de adultos mayores con problemas de memoria, típicos de la edad. Se trata también de que, añadido a ello, se encuentran adultos con experiencias de vida dolorosas que implican un cambio impactante a nivel personal, familiar y social. 
Por todo lo anterior, es necesario trabajar en la promoción de un envejecimiento activo en la población de adultos mayores en el marco del conflicto, con la finalidad que las personas mayores sean autosuficientes en el mayor tiempo de vida posible, sin olvidar la relevancia que tienen las redes de apoyo social en este propósito.

En ocasiones, no solo las personas mayores se sienten como seres ineficaces, lo cual, teniendo en cuenta que se trata de una imagen promovida desde la misma sociedad, obliga a trabajar en el fortalecimiento de las redes de apoyo social, con el fin de contribuir a la inserción social de este grupo poblacional que, de alguna forma, está siendo vulnerado por la misma sociedad. Se entiende por apoyo social a:

Todas aquellas formas de ayuda (material, emocional y de información) que ofrece la sociedad y que los individuos requieren en las diferentes etapas de su vida; se clasifica en: familiar (red primaria), vecinos, amigos o parientes (red secundaria), residan o no con el anciano; y el apoyo institucional o de tipo formal hace referencia a organismos públicos y privados que asisten a la población" (Cardona, Estrada y Agudelo, 2003; citado en Villareal y Month, 2012, p. 76).

Esto es importante tenerlo en cuenta porque las personas adultas mayores que han quedado sin una red de apoyo familiar, son mucho más vulnerables que las personas que cuentan con familiares que pueden cuidar y velar por su bienestar.

La red de apoyo social no solo es importante en la etapa de la edad adulta, sino en todo el ciclo vital con el objetivo de "facilitar el acceso de los colectivos más vulnerables, decisivo para su integración, participación e inserción social con los mismos derechos que el resto de los ciudadanos" (Pérez y Sarrate, 2011; citado en Fernández et al; 2014, p. 537).

Esta red social es fundamental, pues se ha observado que personas mayores sin soporte familiar o con una pared socio familiar inadecuada "tienen mayor mortalidad, depresión, alteraciones cognitivas y una percepción de un peor estado de salud que aquellos que sí lo tienen" (Villareal y Month, 2012, p. 76).

\section{REFERENCIAS}

Bejarano, J., Ardila, L. y Montaño, A. (2014). Alimentación, nutrición y envejecimiento: un análisis desde el enfoque social de derechos. Revista de Facultad de Medicina, 62.

Campo-Arias, A., Oviedo, H., Herazo, E. (2014). Prevalencia de síntomas, posibles casos y trastornos mentales en víctimas del conflicto armado interno en situación de desplazamiento en Colombia: una revisión sistemática". Revista Colombiana de Psiquiatría, 43(4)177-185.
Cardona, D., Estrada, A., Segura, A., Chavarriaga, L., Ordóñez, J. y Osorio, J. (2008) La dependencia del adulto mayor institucionalizado es un asunto de calidad de vida. Revista CES Salud Pública, 62(1), 3-12.

Dueñas, O. (2011). Colombia, España, Uruguay, Costa Rica, Cuba, Ecuador: Seguridad Social para el adulto mayor. Revista de Derecho, 37, 68-110. 
Castillo-Santis et al - Evaluación de la salud mental de personas víctimas del conflicto armado

Fernández, A., García, J. y Pérez G. (2014). Los Programas Universitarios de Mayores y su contribución al aprendizaje a lo largo de la vida. Revista Complutense de Educación, 25(2).

Flecha, A. (2015). Bienestar psicológico subjetivo y personas mayores residentes. Pedagogía Social. Revista Interuniversitaria, 25.

García, J., Fernández, P., Fuentes, L., López, J. y Moreno, M. (2014). Estudio comparativo de dos programas de entrenamiento de la memoria en personas mayores con quejas subjetivas de memoria: un análisis preliminar. Anales de Psicología, 30(1), 337-345.

Hernández-Ramos E, Cansino S. (2011). Envejecimiento y memoria de trabajo: el papel de la complejidad y el tipo de información. Rev Neurol $52,147-53$.

Kilpatrick DG, Resnick HS (2007). Health impact of interpersonal violence. 3: Implications for clinical public policy. J Behav Med., 23(2):79-87.

Ley 1448 de 2011. Por la cual se dictan medidas de atención, asistencia y reparación integral a las víctimas del conflicto armado interno y se dictan otras disposiciones. Recuperado de: http://www.secretariasenado. gov.co/senado/basedoc/ley/2011/ ley 1448 2011.html

Limón, R. y Crespo J. (2001). Ciudad educadora y nuevos espacios de educación para la salud en las personas mayores. Educación, 21(4), 91-123.

López, E. y Giuliani, C. (2012). El examen mental como herramienta diagnóstica en la salud y los trastornos. Eta-Evolutiva, 82, 6773.
Marinês, L., Castioni, D., Kirchner, R. y Hildebrandt, L. (2015). Capacidad funcional y nivel cognitivo de adultos mayores residentes en una comunidad en el sur de Brasil. Revista Electrónica Trimestral de Enfermería, (37).

Melguizo, E., Acosta, A. y Castellano, R. (2012) Factores asociados a la calidad de vida de adultos mayores. Cartagena (Colombia). Salud Uninorte. Barranquilla, 28(2), 251263.

Pousada, M., de la Fuente, J., Gómez, B., y Armadans, I. (2004). Los efectos de la información irrelevante en la memoria operativa de las personas mayores. Revista Multidisciplinar de Gerontología, 14 (1), 16-21

Saunders BE, Kilpatrick DG, Resnick HS,Tidwell RP (2009). Brief screening for lifetime history of criminal victimization at mental health intake: A preliminary study. J Interpers Violence, 4(3), 267-77.

Thornton, W. J. L. y Raz, N. (2006). Aging and the Role of Working Memory Resources in Visuospatial Attention. Aging, Neuropsychology, and Cognition, 13 (1), 36-61

Tonon G. (2010). La utilización de indicadores de calidad de vida para la decisión de políticas públicas. Revista de la Universidad Bolivariana, 9(26), 361-370.

Villarreal, G. y Month, E. (2012). Condición sociofamiliar, asistencial y de funcionalidad del adulto mayor de 65 años en dos comunas de Sincelejo (Colombia). Salud Uninorte Barranquilla, 28(1), 7587. 